\title{
Building waveguides for everyday photonic circuits
}

From mobile phones to computers, the devices we use every day are almost universal in trair use of electricity for through their circuits. However, Dr Richard Hildner at the University of Groningen believes that the capabilities of many modern technologies could improve if they were combined with circuits which operate using light. His team's work has now brought about significant progress towards the characteristics of light wave propagation through specialised materials designed to guide them. o keep our modern civilisation
running smoothly, it is critical for engineers and researchers to design systems which can rapidly and
reliably transmit vast, continual streams of information to different locations. These systems can encompass an enormous range of scales; on the higher end, they include underseas cables which keep entire continents connected to the internet through light-carrying fibre optics. On smaller scales, and far more visible to us, the electrical devices which are now engrained into our everyday lives use electrons to exchang
information between different parts of information between different parts of their constituent circuits, including their processors and memory storage

According to Dr Hildner, this latter approach currently faces a universal problen: since electrons must generate heat as they move through conducting creasingly complex heat managemen processes to avoid overheating. These processes require energy, and so the

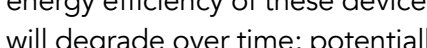
deteriorating their performance turn. The effect is currently severely shortening the lifetimes of many of the technologies we depend on: incuring significant costs for users as they replace their devices more frequen and requiring more devices to be built using manufacturing processes which. are often damaging to the environment. For Dr Hildner and his colleagues, this calls for a fundamental overhaul of the circuits we use to transmit information in modern technologies.

\section{ADVANTAGES OF}

ACTIVE WAVEGUIDES

transmitting light across large

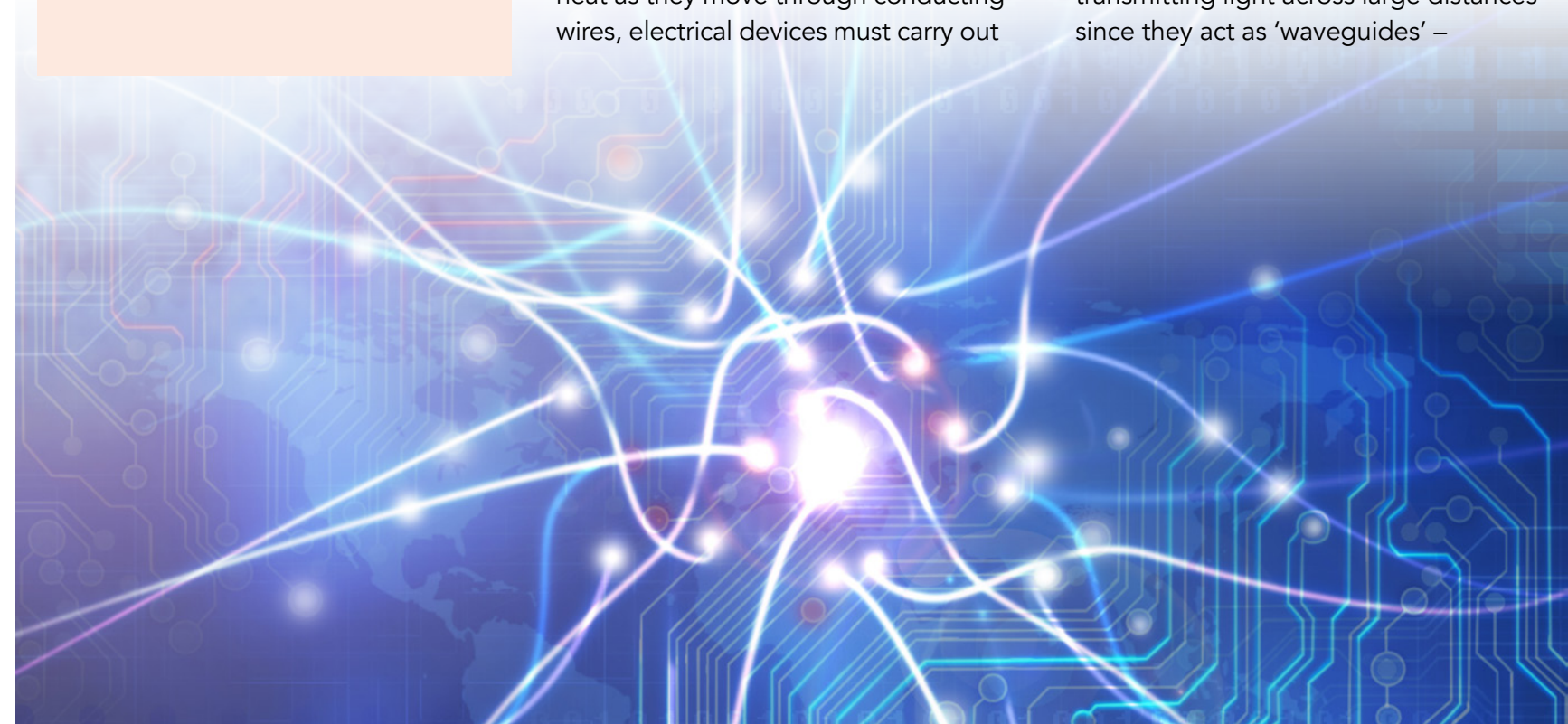

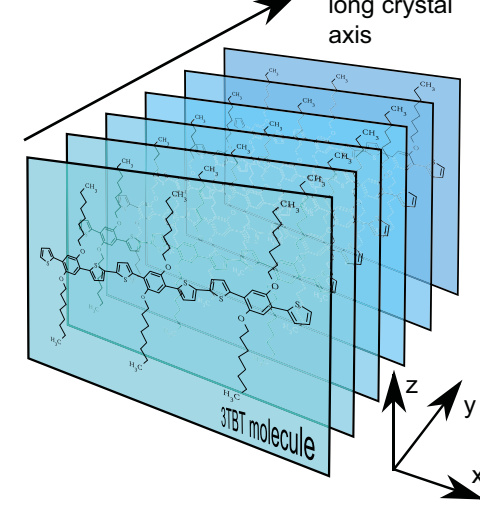

An active waveguide. Unlike their electrical
counterparts, they don't heat up as they op

materials whose elongated geometries can restrict the transmission of light to just one direction, while incurring the righ losses of information. With the right approach, nanostructured transmit light on far smaller scales.

Importantly, however, only light waves with specific frequencies, or wavelengths, are allowed to
travel inside these structures. Depending on their dimensions, particular waveguides will have a series of discrete frequency values named 'modes,' at which light can freely propagate. In many studies today, waveguides provide an important basis for 'photonic circuits, but which don't feature electron-carrying conductive wires. In particular, Dr Hildner's team focus on 'active' waveguides, containing molecules which can themselves em light at specific frequencies, and are tuneable to the waveguide's allowed modes of light propagation. "Since the light to be transmitted in active waveguides is created directly within the waveguide itself, there is no need for an external light source and for complex optics to couple light into waveguides as in today's standard optical fibres," he explains. Ultimately, these materias allow physicists to build compact photonic circuits which, unlike their they operate.

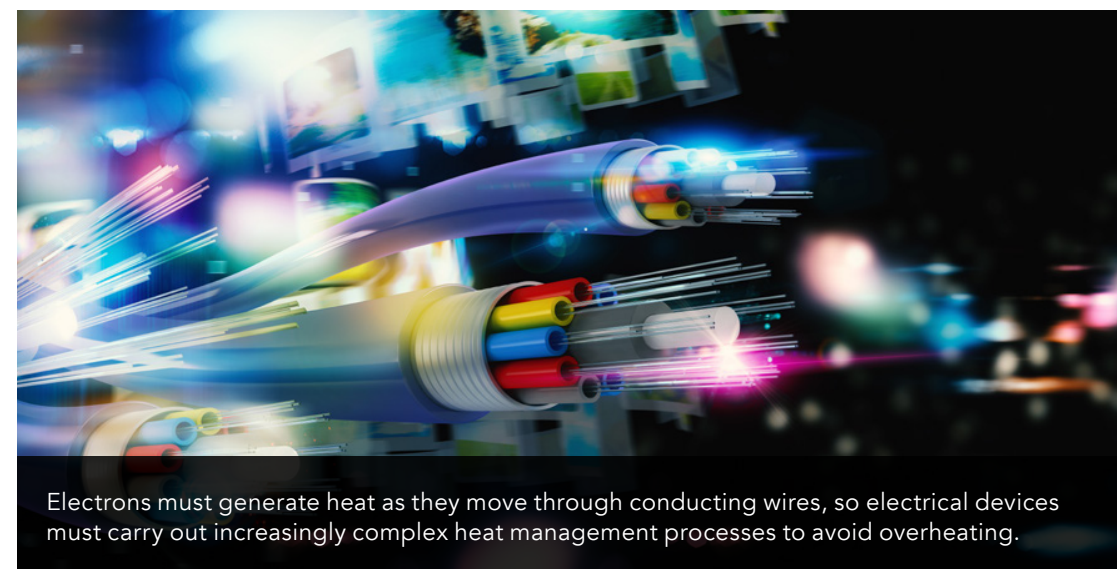

EXPERIMENTS WITH LIGHT-EMITTING WAVEGUIDES In their most recent experiments, Dr hildner and his colleagues investigated how light is transmitted within 'organic single crystals' that form active formed from organic mie crystals are organic molecule used to build these defect-free structures with unique optical to tune the frequency of the light they

Waveguides provide an important that they emit into basis for "photonic circuits" - comparable propagating within to electrical circuits, but which ctive waveguide, their emitted light is largely confined conductive wires.

properties. This property is particularly useful since organic molecules

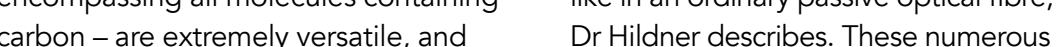
can display a vast array of shapes, sizes, advantages have now been widely and properties. Furthermore, actives studied through theoretical calculations.

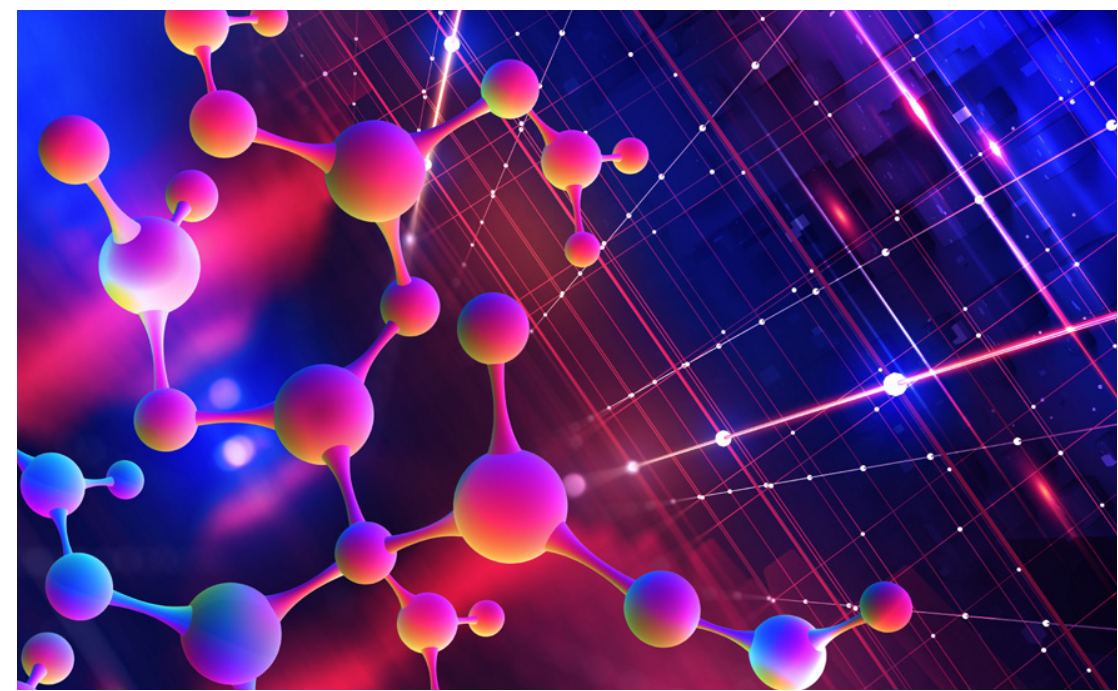




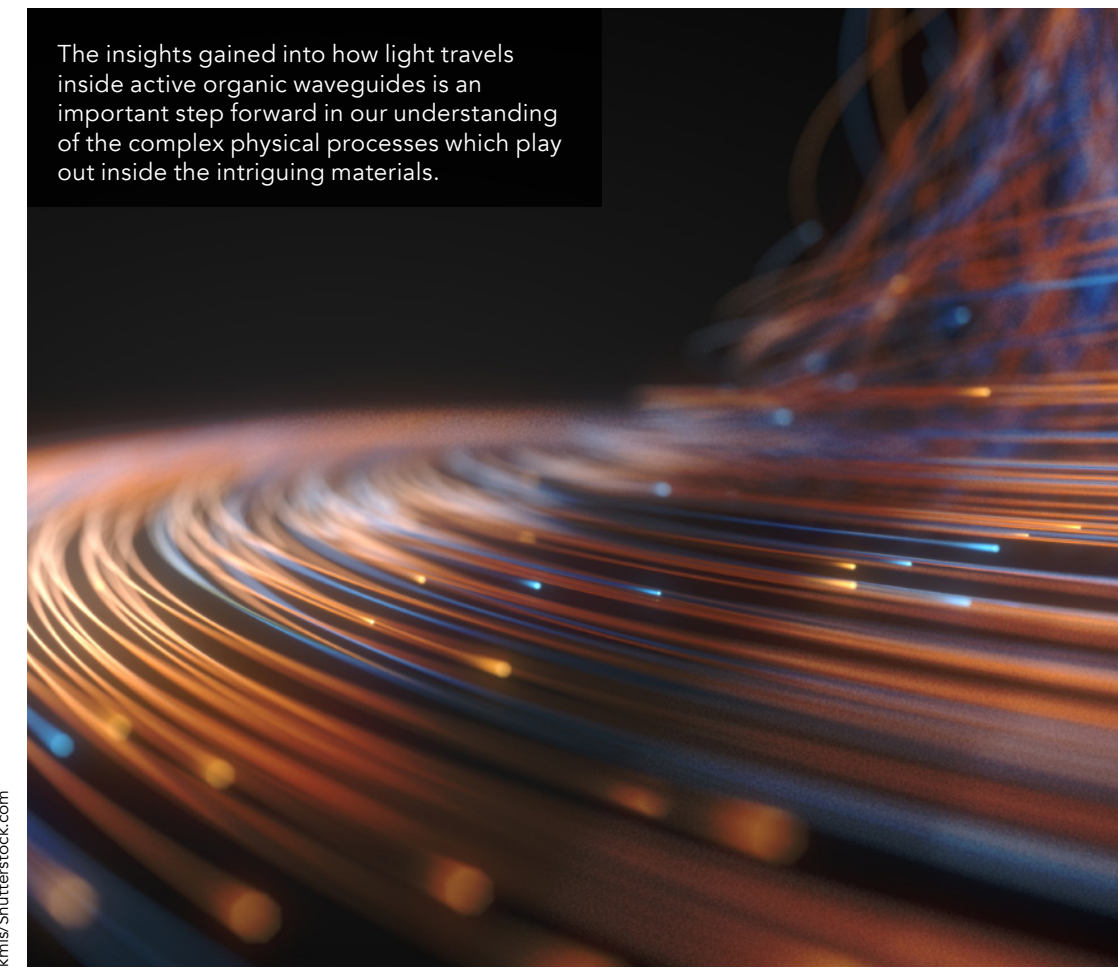

Dr Hildner and his colleagues investigate how light is transmitted within active waveguides based on organic single crystals.

In experiments, however, it is far more difficult to directly measure the physical processes which unfold as light propagates through an active organic waveguide.

\section{INVESTIGATING LIGHT}

TRANSMISSION

Kels to DrSI

index' of organic active wave refractive number relating the speed of light inside a material to its speed in vacuum. Just like more typical transparent materials like glass, the refractive index of these more advanced materials varies with the wavelength of the light passing through it; in other words, with the mode of the light. This variation can be quantified through the so-called 'dispersion

relation,' which can be measured by forcing specific molecules to emit light using a laser.

In these experiments, molecules are first excited to higher energy levels as they interact with the laser. Then, as the
molecule relaxes back into its original brighter overall signal on the detector Alternatively, if they are misaligned, they will be a darker signal than expected from the sum of the original two waves.

For Dr Hildner's experiment, if the positions of the two exit points were varied more widely, the peaks and troughs of each resulting light wave would become more misaligned, increasing destructive interference in turn. Dr Hildner's team measured the differing degrees of constructive and destructive interference in their experiments, thus could determine the degrees to which the organic active waveguide's refractive index varied for the different modes passing through it.

"By measuring, for instance, the constructive interfence pattern as directly reconstruct the dispersion relation of the active waveguide, and have access to this fundamentally important relation for light transmission through media," Dr Hildner concludes. Through further in-depth analysis, the researchers also accounted for factors including the geometry of the waveguide's crystal edges, and the orientation of its constituent molecules. The insights they have gained into how light travels inside active organic waveguides is a fundamentally important step forward in our understanding of the complex physical processes which play out inside
the intriguing materials.

\section{TRANSFORMING}

\section{MODERN CIRCUITS}

Having gained a more in-depth

ght waves propagate Dr Hildner's team are now in a strong position to study how they could be integrated into more complex photonic circuits in the near future. If successful, these efforts could lead to devices which don't overheat as they operate, allowing them to perform well for far longer than their current electrical counterparts. Dr Hildner now hopes to explore the possibility for such applications further in future research; potentially leading to innovations which could transform the capabilities of many technologies which are now important aspects of

\section{Behind the Research}

\section{Dr Richard Hildner}

E: r.m.hildner@rug.nl T: +31503638149 W: https://www.rug.nl/staff/r.m.hildner/

Research Objectives

Richard Hildner's research focuses on optical (singlemolecule) spectroscopy of organic functional molecules

\section{Detail}

Richard Hildner

Zernike Institute for Advanced Materials

University of Groningen

Nijenborgh 4

Bio

in physics from University of Bayreuth in 2008. He has held a position at the Zernike Institute for Advanced Materials (University of Groningen) since 2018.

Funding

\section{Collaborators}

- Crof Gan Schörner, University of Bayreuth, D - DrChristian Neuber, Univeristy of Bayreuth, D

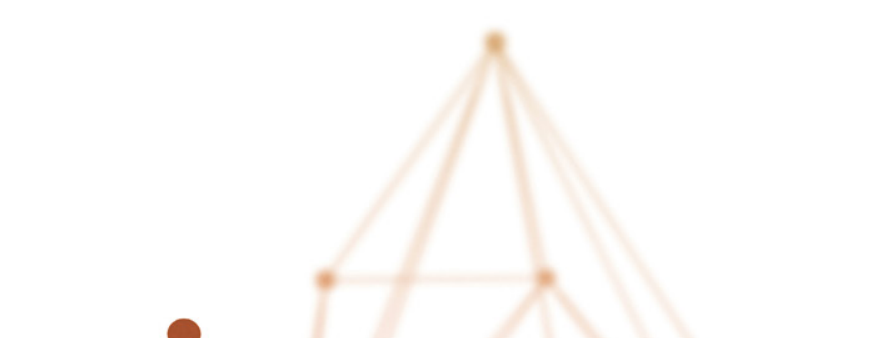

German Research Foundation (DFG)

\section{References}

Schörner, C., Motamen, S., Simon, L., Reiter, G., Hildner, R. (2018). Self-interference of exciton emission in organic single crystals visualized by energy-momentum

Schörner, C. Neuber, C. Hildner, R. (2019). Two-path self-interference in PTCDA active waveguides maps the dispersion and refraction of a single waveguide mode. APL Photonics, 4(1), 016104. https://doi. org/10.1063/1.5068761

\section{Personal Response}

What first sparked your interest in this area of research?

II About a decade ago I got interested in how energy is transported within assemblies of organic molecules, artificial light-harvesting assemblies. We soon realised that in many of those assemblies transport of energy is accompanied by transmission of light, i.e., the assemblies act as active waveguides as well. It became clear that we had to develop a better understanding of
active waveguides and of their properties.
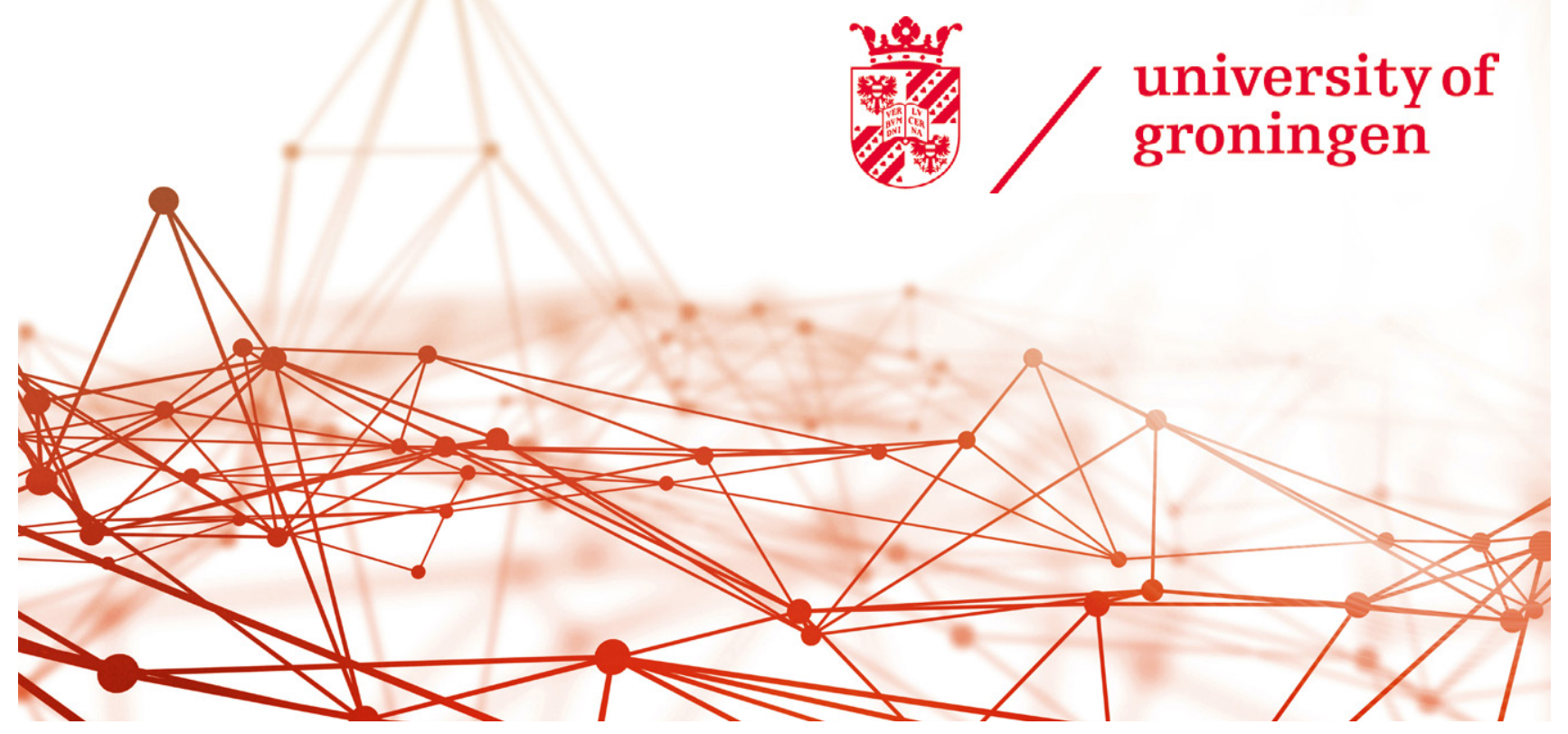